\title{
Development of a new fast shower maximum detector based on micro channel plates photomultipliers (MCP-PMT) as an active element.
}

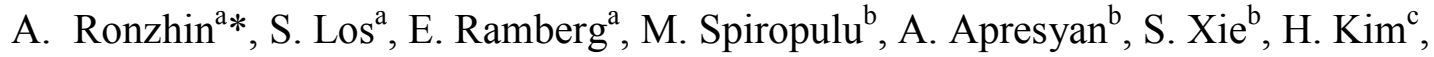
A. Zatserklyaniy ${ }^{\mathrm{d}}$

${ }^{a}$ Fermilab, Batavia, Il 60510, USA

${ }^{b}$ California Institute of Technology, Pasadena, CA, USA

${ }^{c}$ University of Chicago, Chicago, Il 60637, USA

${ }^{d}$ University of California, Santa Cruz, CA, USA

\section{Abstract}

One possibility to make a fast and radiation resistant shower maximum (SM) detector is to use a secondary emitter as an active element. We present below test beam results, obtained with different types of the photo detectors based on micro channel plates (MCP) as the secondary emitter. The SM time resolution - we obtained for this new type of detector is at the level of 20-30 ps. We estimate that a significant contribution to the detector response originates from secondary emission of the MCP. This work can be considered as the first step in building a new type of calorimeter based on this principle.

Keywords

MCP-PMT

SM

DRS4

* Corresponding author. Tel.: 16308408630

E-mail address: ronzhin@fnal.gov (A. Ronzhin) 


\section{I.INTRODUCTION}

The active materials used in SM detectors and calorimeters at high luminosity hadron colliders are scintillators, crystals, quartz fibers, etc. These materials suffer from damage after experiencing high radiation doses and need to be replaced by more radiation resistant materials as integrated luminosity increases. An additional big challenge is to improve the timing properties of the detectors. They should be as fast as possible to operate at high counting rate, and be capable to reduce the background from multiple collisions. The described method could satisfy these requirements.

The first proposal to use secondary emitter materials as an active element in sandwich type calorimeters can be found in the reference [1]. This approach allows the development of a new type of detector that is highly radiation resistant and fast. The measurements with such a type of element were performed with an MCP as an electron multiplier. One of the main limitations to introduce this technique in practice has been the high cost of the electron multiplier. With the recent progress of the Large Area Picosecond Photo Detector collaboration [2], the costs may decrease sufficiently to allow practical construction of these types of detectors.

The principle of the operation of the shower maximum detector (or calorimeter) is the following. High-energy particles passing through absorber material in sandwich type calorimeters produce secondary particles (e.g. positron electrons pairs, gammas, etc.) that are detected by MCPs. The generation of secondary particles in the calorimeter will be referred to as a "shower". The response of the MCPs to the secondary particles is proportional to their number, which is in turn proportional to the energy of the initial particle.

Most of the secondary particles have low energy, where the MCP's detection efficiency is high. The data on the low energy component of the particle showers are not well investigated, and even reliable Monte Carlo simulation is limited by the minimal energy of the secondary particles to $\sim 100 \mathrm{KeV}$. Therefore, to explore the feasibility of a calorimetric device based on MCP's as the secondary emitter we started with experimental measurements. The results are presented in this paper. 


\section{DESCRIPTION OF THE PHOTO TUBES.}

We used two types of photo detectors based on MCPs. One of them was a Photek 240. The external view and some dimensions of the Photek 240 are presented in Fig. $1(a, b)$.

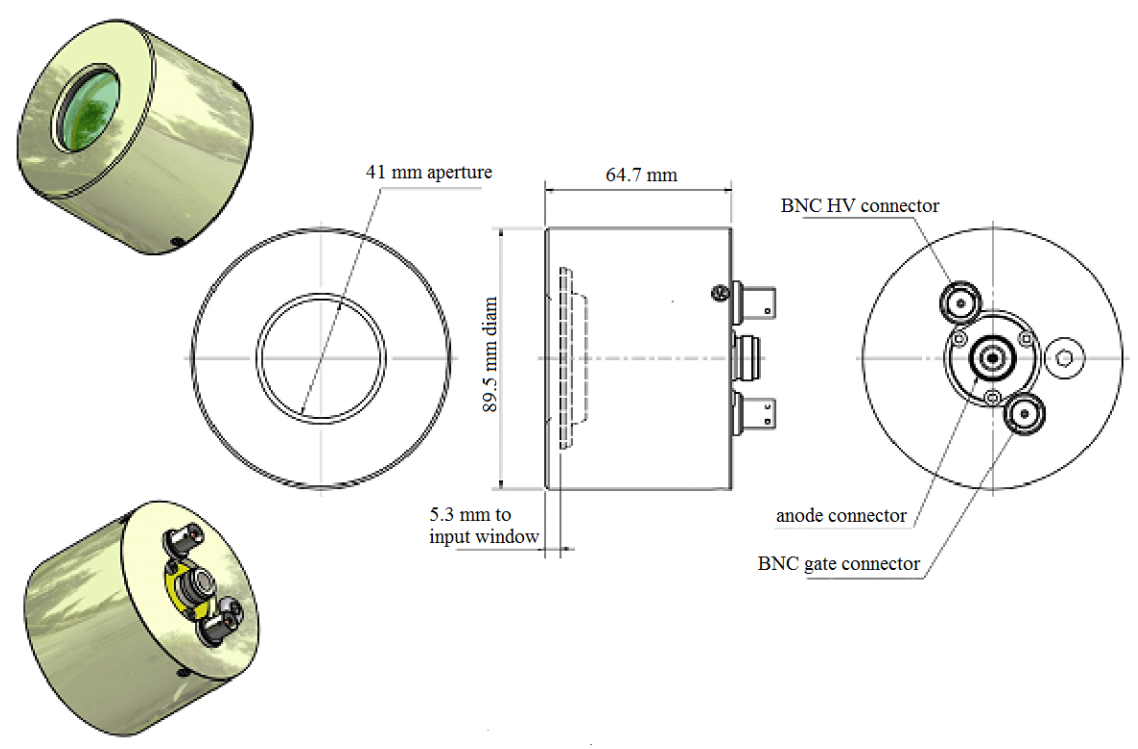

Fig.1 (a). The external view and some dimensions of the Photek 240.

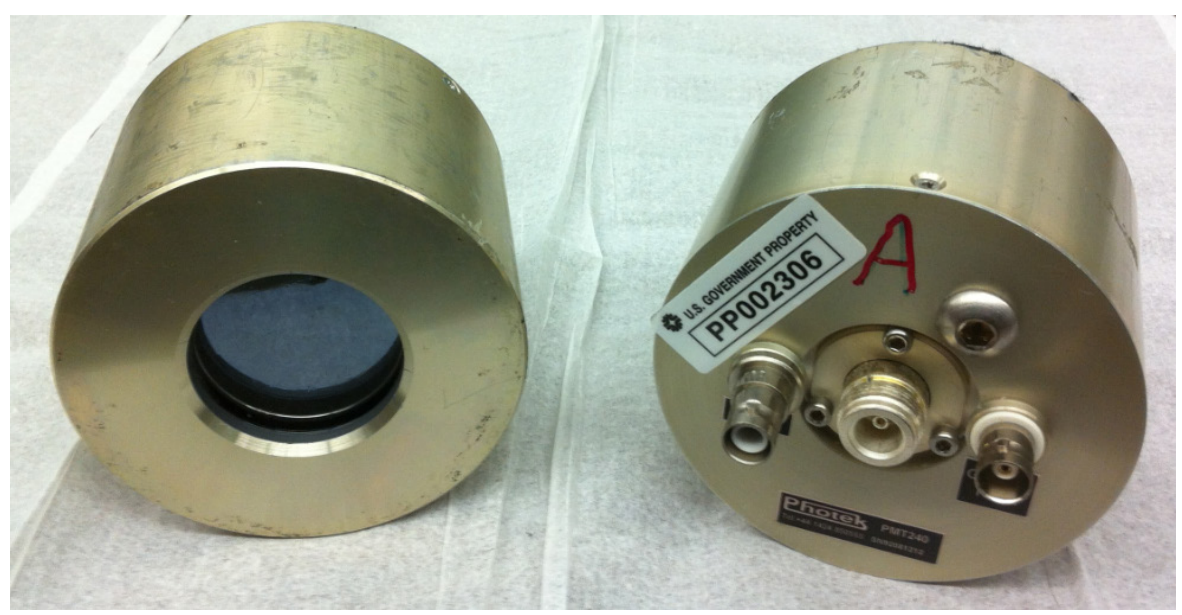

Fig.1 (b). Picture of the two Photek 240 tubes, used in this work.

Main parameters of the Photek 240 were measured and presented in [3]. An MCP consists of a parallel array of thin microtubules containing an emissive layer for electron amplification. The pore size is on the order of $10 \mu \mathrm{m}$ and the distance from the photocathode to the first amplification stage is only a few $\mathrm{mm}$. The 
Photek 240 has a $41 \mathrm{~mm}$ circular sensitive area. The single photoelectron time resolution (SPTR) was measured to be $\sim 40$ ps [3]. The output signal time shift is limited to $\sim 3.9$ ps across the sensitive area of the Photek 240 . The input window of the phototube made of $9 \mathrm{~mm}$ thick quartz which enables extended UV quantum efficiency. The high voltage (HV), applied to the Photek 240 is $\sim 4.8 \mathrm{kV}$. We used Bertan model $380 \mathrm{~N}$ unit as the HV power supply. The output pulse shape from the Photek 240 illuminated by a PiLas laser light is presented in Fig. 2. The PiLas laser light pulse duration is 33 ps (FWHM).

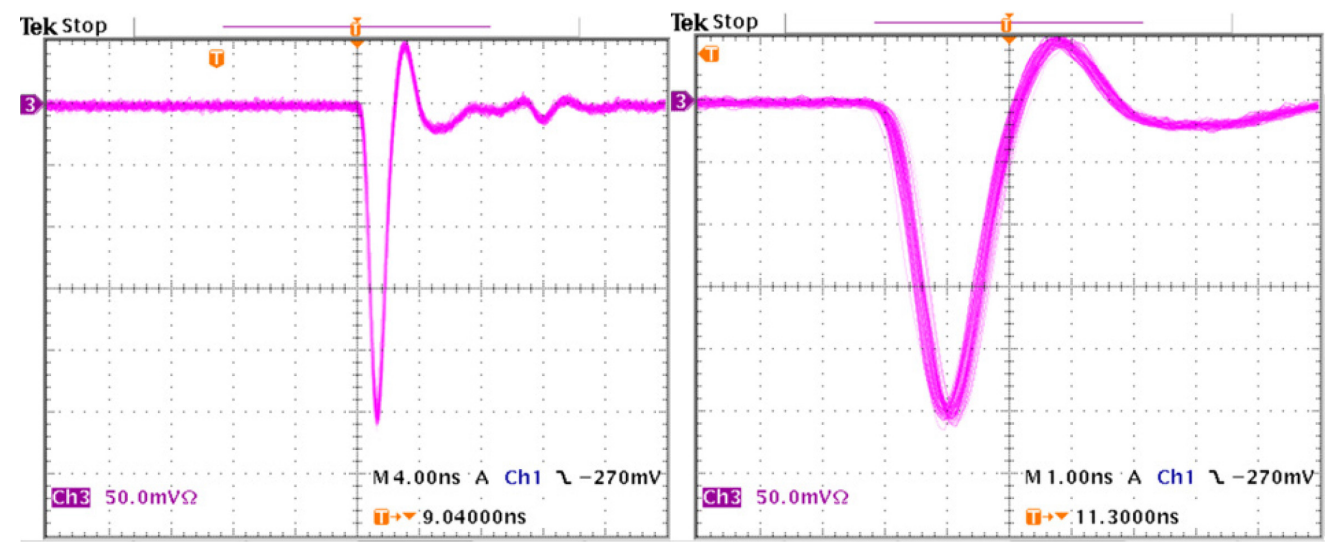

Fig. 2. Output pulse shape of the Photek 240, measured by Tektronix oscilloscope (TDS 3054B, $500 \mathrm{MHz}, 5 \mathrm{GS} / \mathrm{s}$ ). 4ns/div - left, $1 \mathrm{~ns} / \mathrm{div}$ - right.

The other MCP-PMT was Photonis 8501 (Fig. 3). One advantage of the Photonis MCP-PMT is its large sensitive area, which is similar to standard PMTs.

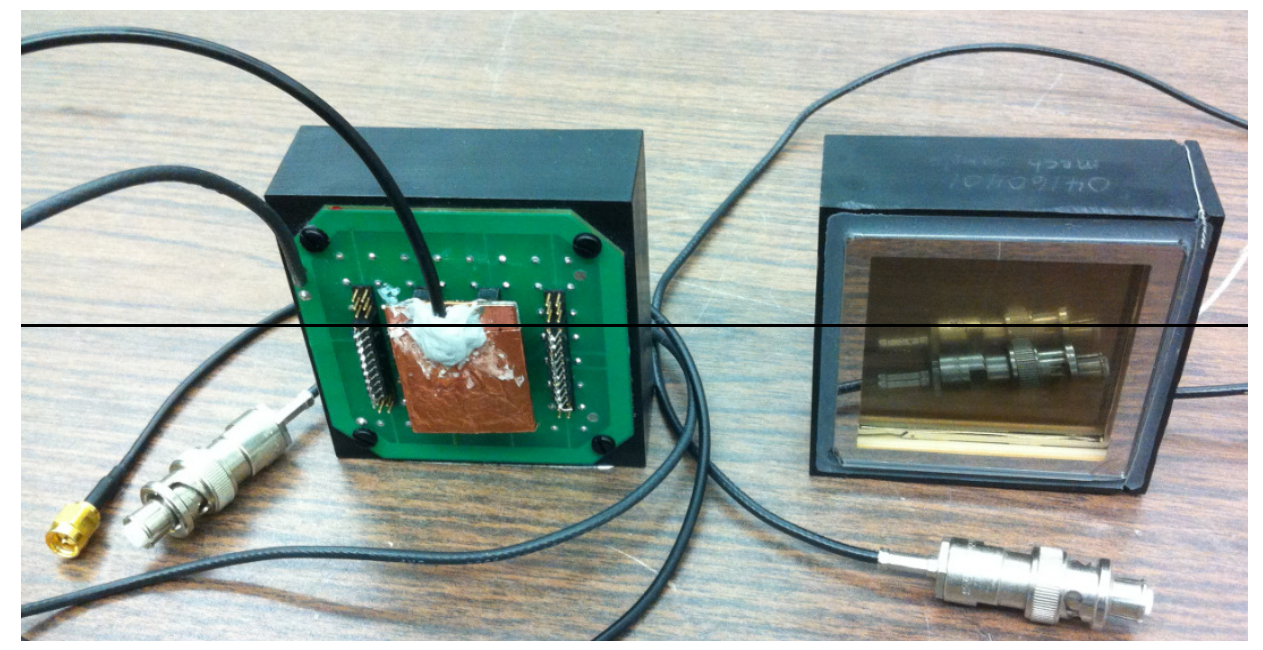

Fig. 3. Picture of the Photonis 85011 (front and rear view). Only one was used in this work. 
The anode of the Photonis 85011 MCP-PMT is composed of 64 pads, arranged as an $8 \times 8$ matrix [4]. The size of each pad is $6 \times 6 \mathrm{~mm}^{2} .16$ central pads ( $4 \times 4$ matrix) were connected together making a sensitive area of $\sim 24 \times 24 \mathrm{~mm}^{2}$. The input window is made of UV Grade Fused Silica with a thickness of $2 \mathrm{~mm}$. The tube has a bi-alkali photocathode with maximum quantum efficiency of about $23 \%$ at $400 \mathrm{~nm}$. The pore size of the Photonis MCP-PMT is $25 \mathrm{um}$. The signal rise time is $\sim 0.3 \mathrm{~ns}$, and the anode pulse width (FWHM) is $1.8 \mathrm{~ns}$. We measured a SPTR of $\sim 120$ ps with low gain Photonis MCP-PMT [5]. Timing non-uniformity along the photo cathode is $\sim 37$ ps when using the passive sum of the 16 pads. The value was measured by illuminating $\sim 1 \mathrm{~mm}$ diameter spot across the photocathode and measuring the time interval between the Pilas laser trigger and the Photonis output signal. We used electronics based on Ortec units for the measurement [3]. The HV applied to the tube was $2.4 \mathrm{kV}$, with a corresponding gain of $\sim 10^{6}$. Fig. 4 presents a cross section of the Photonis MCP PMT and some mechanical dimensions.

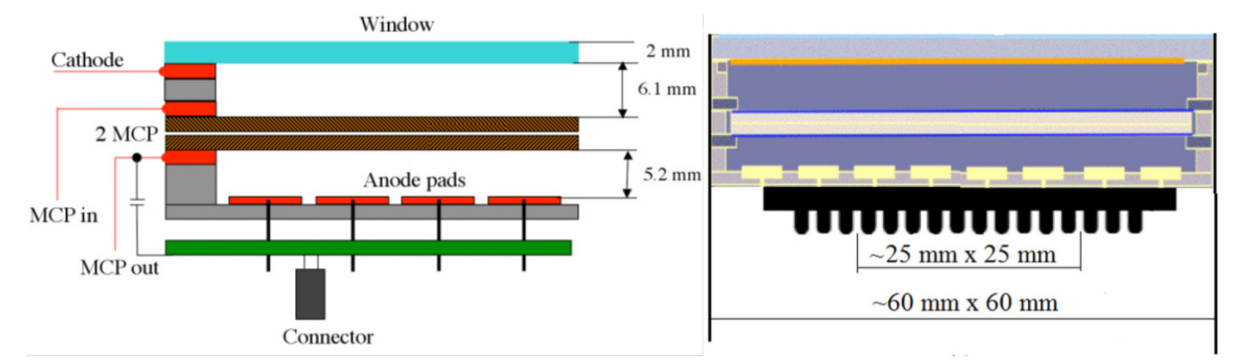

Fig. 4. Cross-section of the Photonis MCP-PMT, some dimensions.

The output signal of the Photonis is shown in Fig. 5. The tube was illuminated by the PiLas laser. Some ripples are seen after the signal (Fig. 5). An effect is due to tying multiple anodes together.

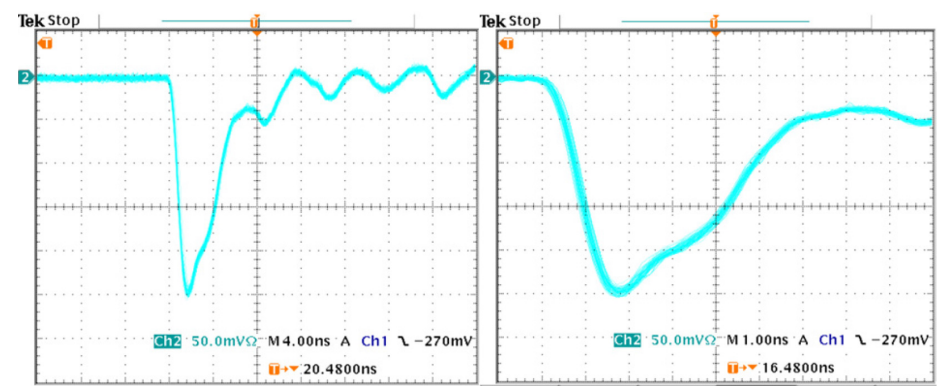

Fig. 5. Output signal of the Photonis MCP-PMT, taken by the Tektronix oscilloscope (TDS 3054B, $500 \mathrm{MHz}, 5 \mathrm{GS} / \mathrm{s}$ ). $4 \mathrm{~ns} / \mathrm{div}$ - left, $1 \mathrm{~ns} / \mathrm{div}$ - right. The area corresponding to the 16 central pads is illuminated by PiLas laser light. 


\section{TEST BEAM SETUP.}

We used the Fermilab Test Beam Facility (FTBF), which provided proton beams from Fermilab's Main Injector accelerator at $120 \mathrm{GeV} / \mathrm{c}$, as well as secondary positron beams with $12 \mathrm{GeV} / \mathrm{c}$ and $32 \mathrm{GeV} / \mathrm{c}$. Detectors were located inside of a dark box lined with copper foil for RF shielding (Fig. 6). Trigger was based on a 10x10 $\mathrm{mm}^{2}$ scintillation counter. The three detectors (two Photek 240 and one Photonis between them) were placed in line. A stack of lead generating a shower, when high energy particle pass through it, was placed before MCP-PMT (Photonis in this case, Fig. 3). The Photonis detector was swapped by second Photek 240 in some of the measurements, without any modification to the cabling. We name the detectors below as "start" counter (always first Photek 240), upstream (Photonis in the Fig. 3) and downstream shower maximum (SM) detectors.

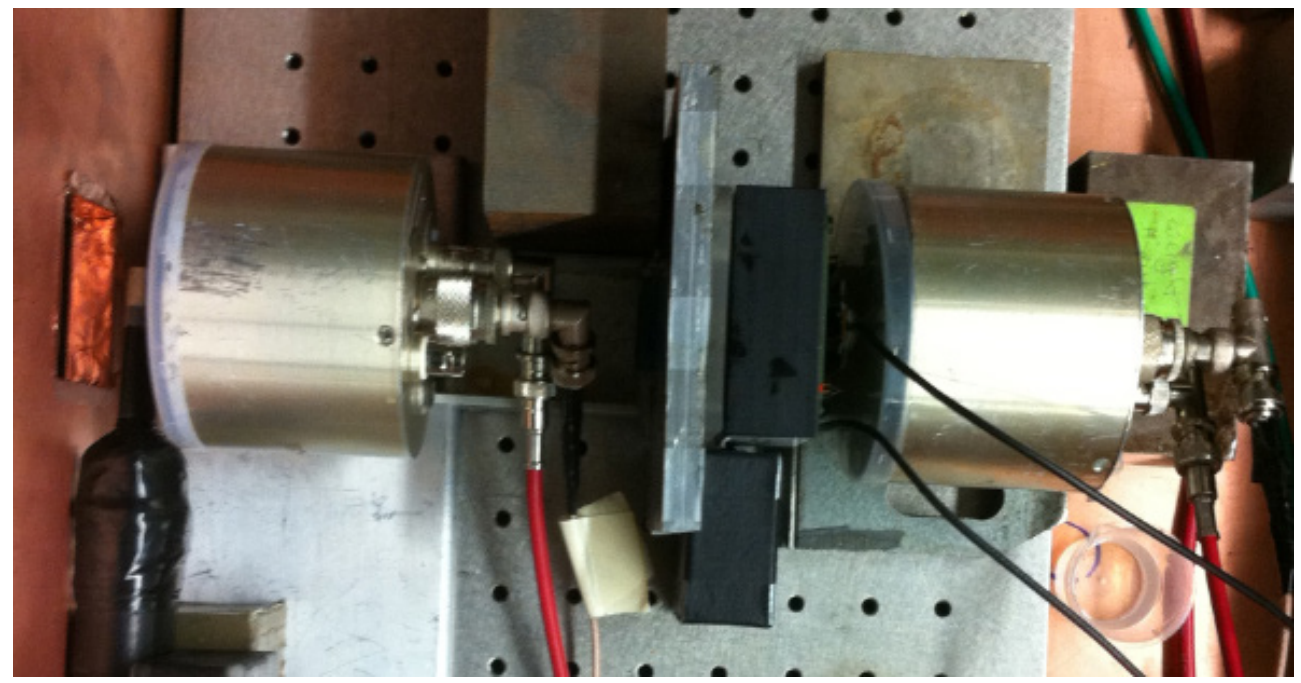

Fig. 6. Part of the test beam setup. Starting from left: Photek 240 ("start" counter), upstream Photonis 85011 and downstream Photek 240 were placed on the optical table (for normal particle incidence). A stack of lead plates can be seen to the left from Photonis detector. A Cherenkov counter was located before the dark box (in the beam path, not shown in the picture).

Two DRS4 waveform digitizer units [6] performed the main readout. The DRS4 were triggered by TTL level signals originating from the trigger counter. Signals from the four detectors, two Photek 240, the Photonis and the Cherenkov counter were split by high frequency Mini-Circuits ZFRSC-42-S+ splitters 
(4.2GHz BW). The outputs were connected in the same order to two DRS4 units. We attenuated the input signals to one of the two DRS4 units to cover the full dynamic range of the measurements. The other DRS4 did not have any additional attenuation. The schematic of the readout with the DRS4 units is shown in Fig. 7.

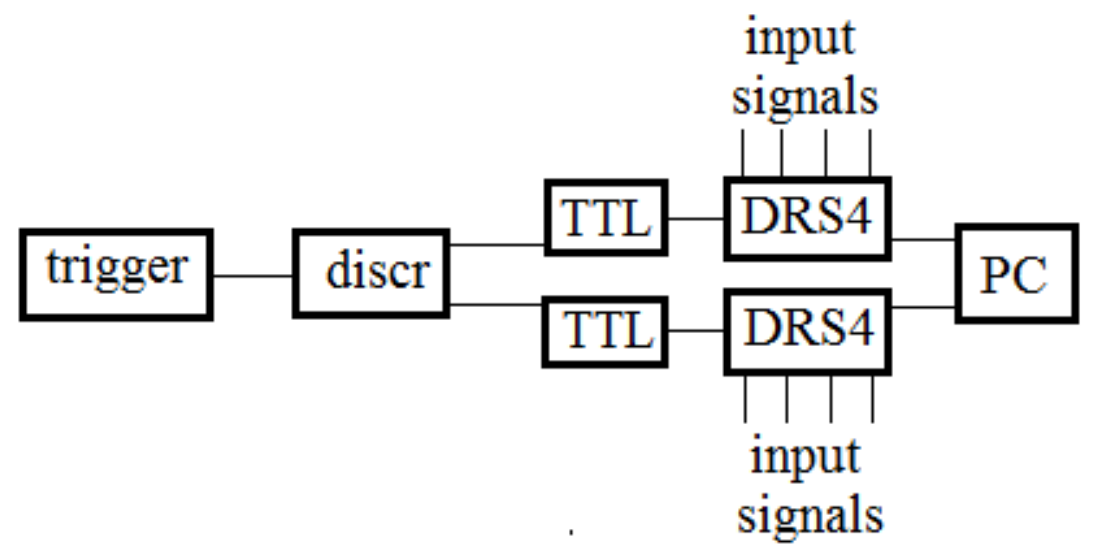

Fig. 7. Schematic of the readout: discr: - LRS 621 BL (quad discriminator), TTL: - LeCroy 223 unit, PC - readout computer.

\section{EVENT SELECTION AND ANALYSIS}

To assign a time stamp for each signal pulse, we first determine the time position of the pulse peak. A Gaussian function is fitted to the pulse maximum using three points before the maximum of the pulse peak and four points after the maximum. The mean value of the Gaussian was used as the time stamp for each pulse. Event selection and pulse cleaning procedure eliminated abnormal pulses in the readout. Large signals above $500 \mathrm{mV}$ were also rejected because they saturated the DRS4 inputs. Pulses with an irregular peak profile were rejected, as well as pulses which experienced a sudden reversal of polarity that is occasionally observed with our readout. We selected the pulses with larger than $20 \mathrm{mV}$ amplitude for future analysis. Events containing more than one pulse within our readout window were not used.

For the Photonis detector, we observed a linear dependence of the measured time difference between signals of the "start" counter (Photek 240, channel 1) and the Photonis (channel 3) with the amplitude of the Photonis detector signal, as shown in Fig. 8. Therefore we perform a time correction for each event on the measured amplitude. 


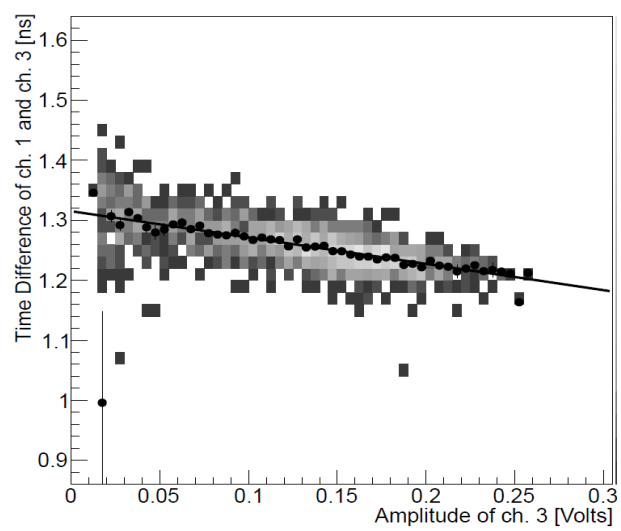

Fig. 8. Dependence of the time difference between the channels 1 and 3 on the signal amplitude in channel 3, (rectangular points). The black circles present the mean values. Linear fit applied for the mean values.

\section{TEST BEAM MEASUREMENTS}

First we measured the "electronic" time resolution of the DRS4 units. The signal from one of the Photek 240 detectors was split into two equal parts, and connected to adjacent channels on the same DRS4 unit. The distribution of the time difference between these two channels was measured, and fitted with a Gaussian. The measured electronic time resolutions of the two DRS4 units were slightly different. The result was dependent on the signal amplitude and was $\sim 4.8$ ps (sigma, Gaussian fit) for one DRS4 and $\sim 6.7$ ps for the other one at the same signal amplitude. We attribute the difference to the higher input noise in one of the two DRS4 units, which was an earlier version of the device.

Distribution illustrating electronic time resolution of the DRS4 is shown in Fig. 9.

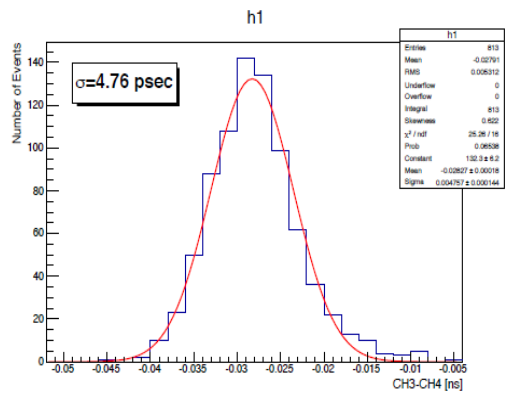

Fig. 9. Distribution presenting electronic time resolution of the DRS4, and a Gaussian fit for it, obtained for the test beam setup. 
The time-of-flight resolution, obtained with $120 \mathrm{GeV} / \mathrm{c}$ protons for the two Photek 240 was $\sim 16.6$ ps. We present our results in sigma of fitting Gaussian distribution everywhere below. Fig. 10 shows an example of such a spectrum. Our previous result for the TR was 12.4 ps [3], but it was obtained in a different setup. In the current measurements the signal of the first Photek 240 was always used as "start". We did not make any correction of the obtained TR to account for the electronic time resolution, which contributes no more than $10 \%$ into the measured TR. The signal of the "stop" counter was taken from the second in line MCPPMT. The lead was placed before this counter.

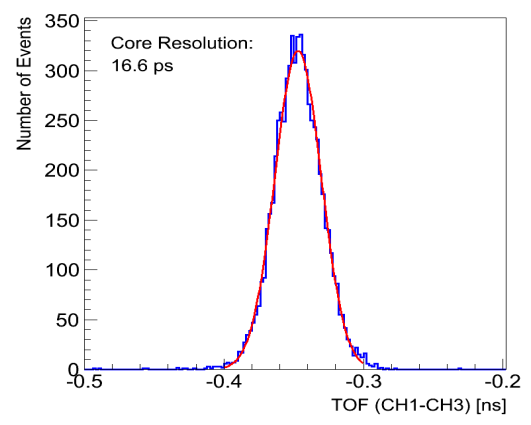

Fig. 10. Spectrum presenting time resolution for the two Photek 240 in line, obtained on the $120 \mathrm{GeV} / \mathrm{c}$ proton beam. The timing delay between the start and the stop signals was chosen close to $0 \mathrm{~ns}$.

The Photek 240 signal from $120 \mathrm{GeV} / \mathrm{c}$ proton recorded by the DRS4 is shown in Fig. 11. This signal perfectly reproduces pulse shape obtained with the PiLas laser in the bench setup (see Fig. 2 above).

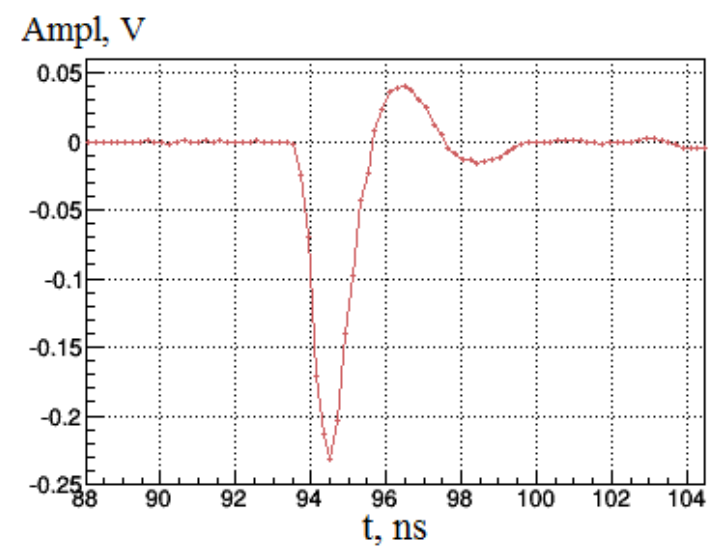

Fig. 11. Signal of the Photek 240 recorded with a DRS4 during a run with $120 \mathrm{GeV} / \mathrm{c}$ protons passing through the PMT input window. 
Next measurements were performed with a positive $12 \mathrm{GeV} / \mathrm{c}$ secondary beam. We refer to these measurements as SM below. The goal of the measurements was to measure the dependence of the detector signal amplitude and time resolution on the lead thickness. We made some measurements with different quartz plates installed in optical contact with the Photonis detector input window to estimate the contribution of Cherenkov light into the MCP-PMT response. The plates were made of UV Grade Fused Silica with the thickness of $3.5 \mathrm{~mm}$ and 7 $\mathrm{mm}$. The transverse size of the plates was the same as that of the Photonis window $\left(\sim 50 \times 50 \mathrm{~mm}^{2}\right)$. The stack of the lead plates with total thickness of either $10 \mathrm{~mm}$ or $20 \mathrm{~mm}$ was placed close to the Photonis input window. The transverse size of each set of the lead well covered the transverse size of the MCP-PMT. For measurements with the positive secondary beams, we selected positron events with the Test Beam Facility's - gas Cherenkov counter. The fraction of the positrons in the $12 \mathrm{GeV} / \mathrm{c}$ positive secondary beam was $\sim 50 \%$.

We found that it is also possible to identify positrons using the signal of the downstream Photek 240. For positron events large signals near the end of the dynamic range of the unattenuated channel were produced. Using positron events selected by the gas Cherenkov counter we found the efficiency of the amplitude selection with the downstream Photek 240 to be $\sim 97 \%$. We have selected positrons with Cherenkov counter with $12 \mathrm{GeV} / \mathrm{c}$ beam and downstream SM with $32 \mathrm{GeV} / \mathrm{c}$. We present the pulse height ( $\mathrm{PH}$, attenuated channel) and time distribution for 10 $\mathrm{mm}$ of lead placed upstream of the Photonis in Fig. 12.
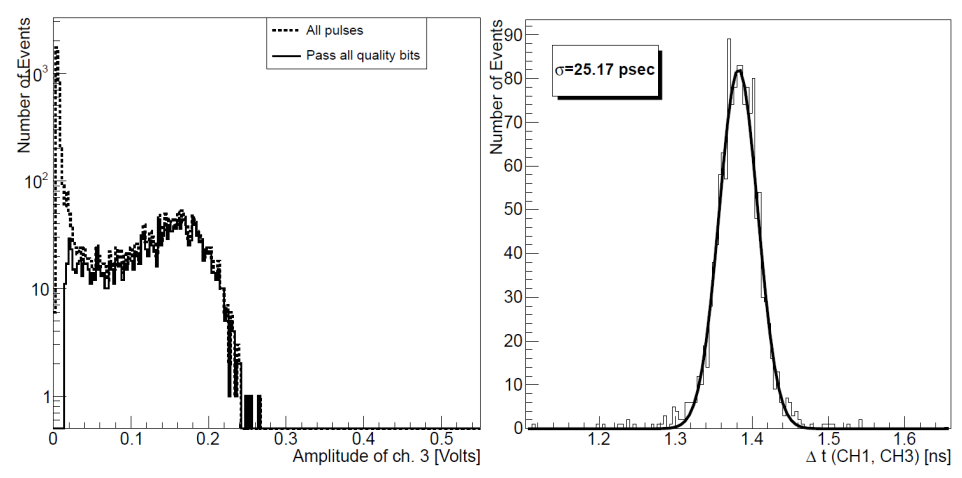

Fig. 12. Pulse height distribution, at the left. Distribution of the time difference between signals of the start Photek 240 and Photonis, at the right. Conditions: $12 \mathrm{GeV} / \mathrm{c}, 7 \mathrm{~mm}$ of quartz plate optically attached to window, $10 \mathrm{~mm}$ of lead before the Photonis. 
The spectra corresponding to zero and $3.5 \mathrm{~mm}$ quartz plates thickness are shown in Fig. 13.
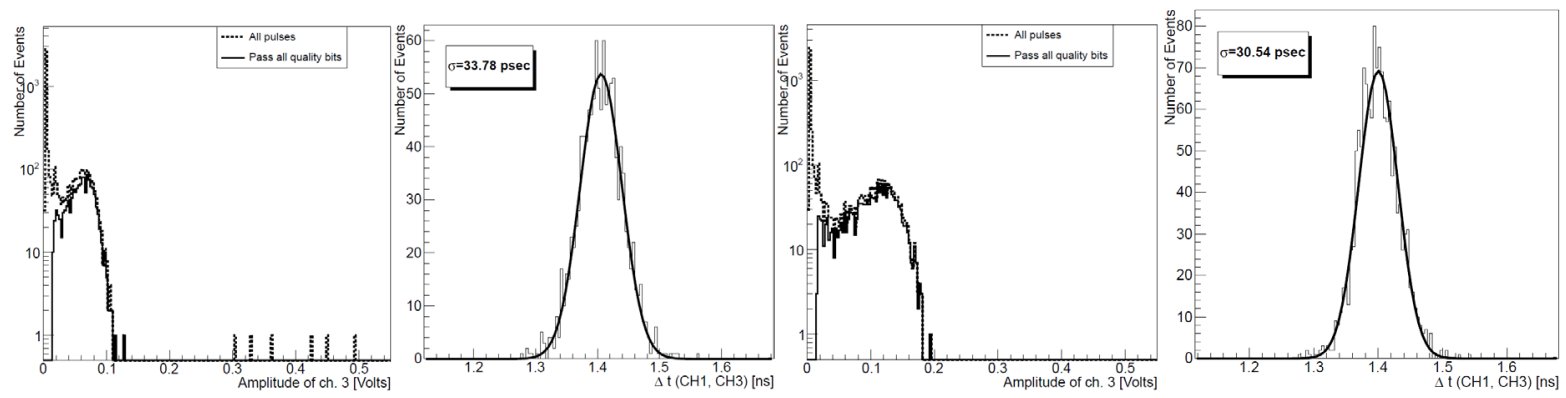

Fig. 13. Distributions of the Photonis pulse height and time difference between the start Photek 240 and Photonis. Same conditions as in Fig. 12. The difference is that no quartz plate was attached to the Photonis window, two pictures at left, and a $3.5 \mathrm{~mm}$ quartz plate was attached to the Photonis window, two right pictures.

We can see a clear peak corresponding to the SM in the pulse height $(\mathrm{PH})$ distributions (Fig. 13), even without any additional quartz attached to the Photonis input window. The mean value of the $\mathrm{PH}$ distribution was calculated.

Fig. 14 shows dependences of the $\mathrm{PH}$ and TR distributions on the quartz plate's thickness.

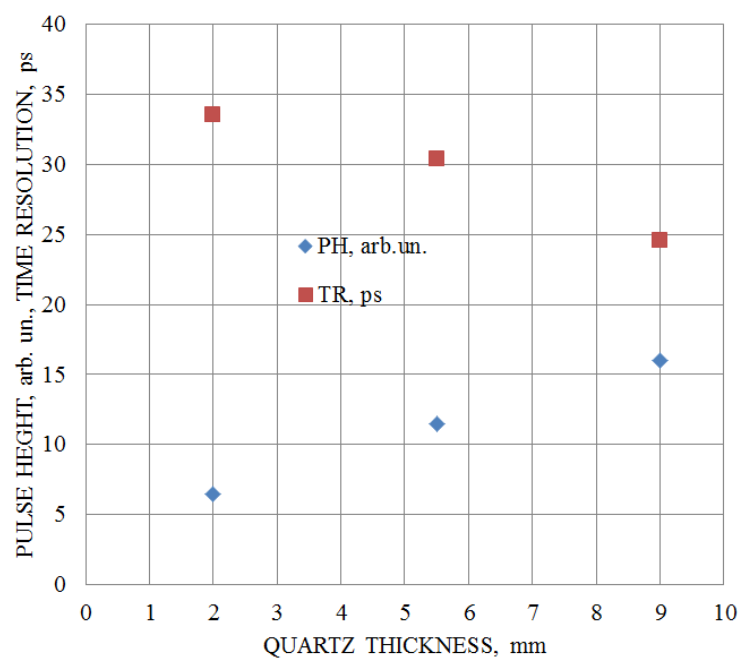

Fig. 14. Dependences of the mean values of the PH and TR distributions on the quartz plate's thickness. Conditions: Photonis as SM, $12 \mathrm{GeV} / \mathrm{c}, 10 \mathrm{~mm}$ of lead before the Photonis. The data points are taken from the Figs. 12, 13. 
Fig. 15 presents data for PH and TR for the Photonis without quartz attached to its window, without lead and with $20 \mathrm{~mm}$ lead thickness.
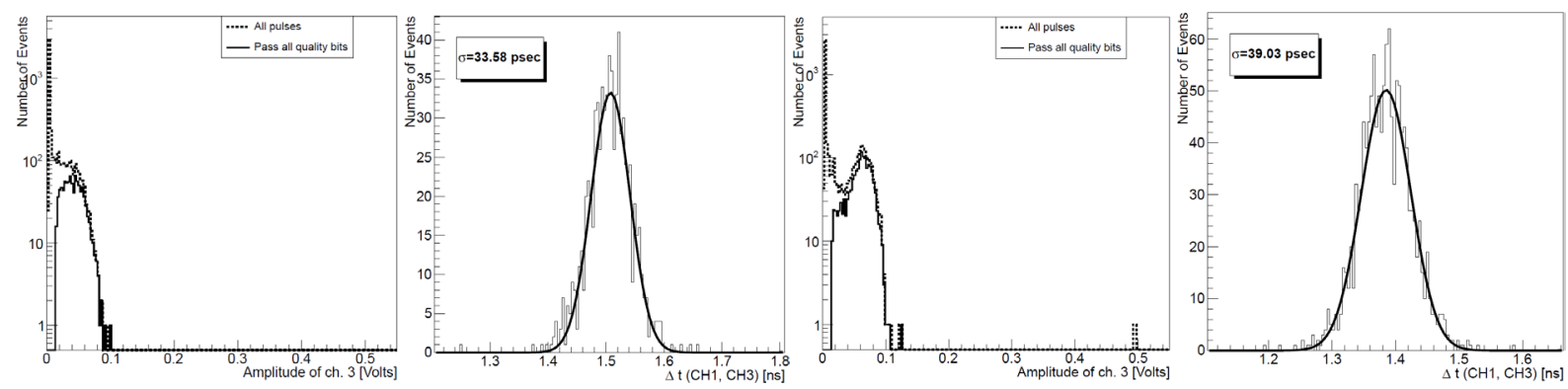

Fig. 15. Distributions of the Photonis pulse height and time difference between the upstream Photek 240 and the Photonis as SM. Conditions: $12 \mathrm{GeV} / \mathrm{c}$, two left pictures - no lead, two right pictures - lead, $20 \mathrm{~mm}$.

Fig.16 shows the PH and TR dependences on absorber thickness in radiation lengths units. No additional quartz was added to the Photonis window. The amount of radiation lengths quoted in Fig. 16. includes the material of the upstream Photek 240 ("start" counter), which is in the beam path.

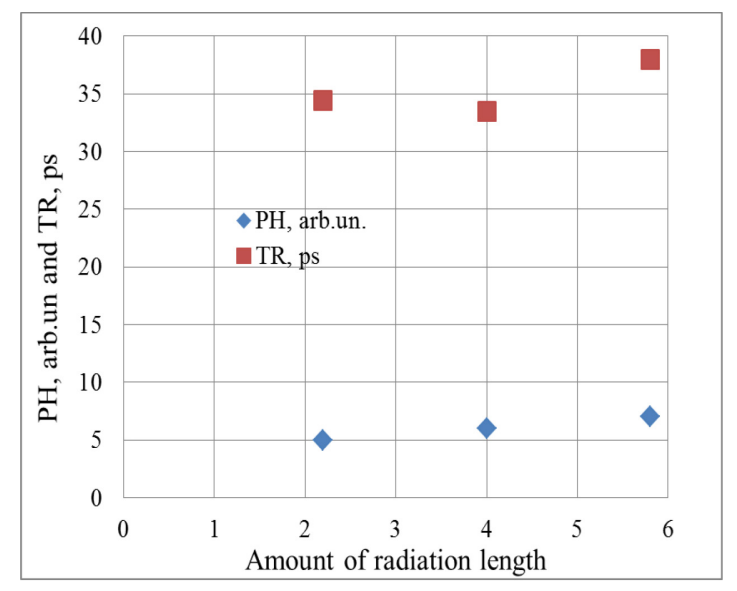

Fig.16. PH and TR dependences on absorber thickness in radiation length units. The data points are taken from Fig. 15. Photonis as SM, beam momentum is $12 \mathrm{GeV} / \mathrm{c}$.

The obtained results for $\mathrm{PH}$ distributions and timing spectra for different detectors were dependent on the procedure used for the events selection. These procedures and criteria are described in part IV above. The same measurements were performed with Photek 240 as the SM detector in the $12 \mathrm{GeV} / \mathrm{c}$ secondary 
beam. No additional quartz was attached to the input window. We measured TR of the SM as a function of the lead thickness. The obtained TR values equal to 27.3 ps, $22.2 \mathrm{ps}$ and $23.5 \mathrm{ps}$ for the lead thickness of zero mm, $10 \mathrm{~mm}$ and $20 \mathrm{~mm}$, Fig. 17. The PH distributions from the SM are not shown since they saturated the DRS4 inputs (even with used attenuators in this measurement).

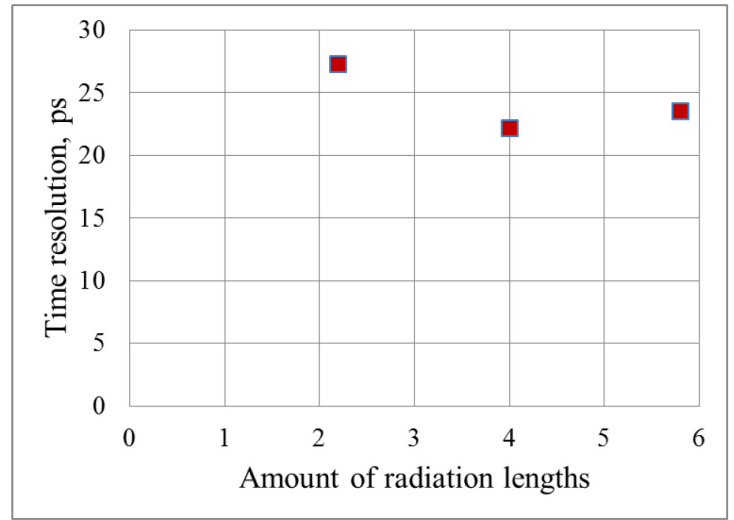

Fig. 17. Dependence of the TR on the absorber thickness (in units of radiation length). Photek 240 as $\mathrm{SM}, 12 \mathrm{GeV} / \mathrm{c}$ positrons.

The next series of measurements with the SM was performed at $32 \mathrm{GeV} / \mathrm{c}$. We used the downstream shower maximum detector (Photek 240) to select positrons with the $32 \mathrm{GeV} / \mathrm{c}$ beam. According to our estimation the positrons component in the beam was less than $10 \%$. We carried out the measurements with $10 \mathrm{~mm}, 20 \mathrm{~mm}$ of lead thickness and without lead. The TR dependence on absorber length is shown in Fig. 18.

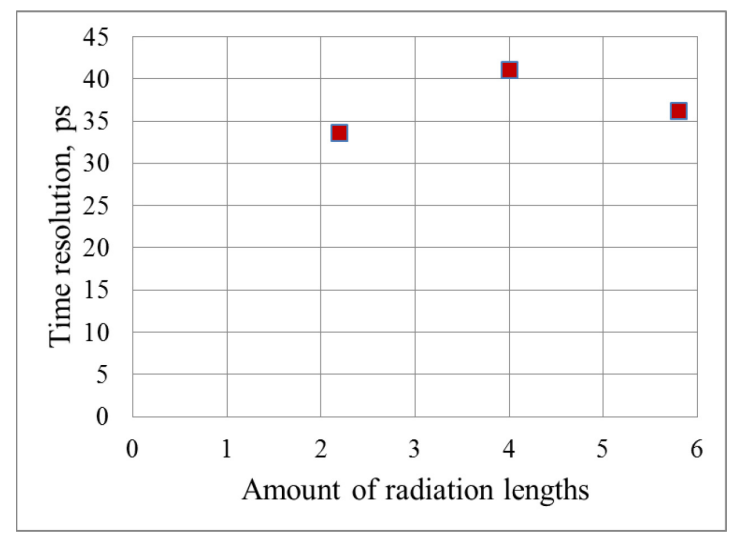

Fig. 18. Dependence of the TR on the absorber thickness (in units of radiation length). Conditions: Photonis as SM. Beam momentum is $32 \mathrm{GeV} / \mathrm{c}$; lead thickness: $20 \mathrm{~mm}$, left, $10 \mathrm{~mm}$, middle, $0 \mathrm{~mm}$, right, before the Photonis. 
Fig. 19 shows the dependence of the TR on the absorber thickness in the amount of radiation length obtained with the Photek 240 as SM. The beam momentum is $32 \mathrm{GeV} / \mathrm{c}$. We still observe the TR at the level of the $25-30 \mathrm{ps}$.

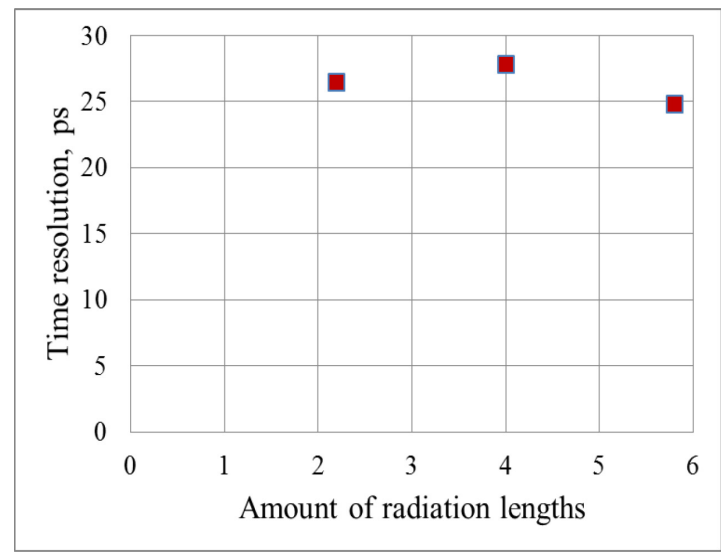

Fig. 19. Dependence of the TR on the absorber thickness. Conditions: Photek 240 as SM, $32 \mathrm{GeV} / \mathrm{c}$ positrons.

\section{DISCUSSION}

The obtained data indicated that the shower particles were registered through the Cherenkov radiation in the input window as well as through direct interaction with the MCP. The secondary emission component of the response looks significant. Under the assumption that the Cherenkov response increases linearly with the radiator thickness (Fig. 14), one can extrapolate the dependence of the $\mathrm{PH}$ on the thickness of the Photonis input window down to zero to get an estimate for the secondary emission. We can estimate that $\sim 70 \%$ of the MCP-PMT response is due to the MCP secondary emission and $30 \%$ is due to Cherenkov light in the 2 mm thick input window. The Cherenkov light produced by shower's particle hitting the window. The TR of the secondary emission component can be estimated to be around 35-40 ps for the Photonis MCP-PMT.

The Photek 240 shows an even better SM TR, $\sim 20-30$ ps. The Photek 240 MCP-PMT has an input window thickness of $9 \mathrm{~mm}$. We did not make measurement with different input window thickness, but we have seen the response increase with additional radiator optically coupled with the Photek 240 input window. Nevertheless we can assume that the low energy component of the shower detected by an MCP will be significant, no matter which device is used. 
We can compare the TR results obtained for the Photek and Photonis detectors with the same input window thickness, $9 \mathrm{~mm}$. We attribute the better results obtained with the Photek 240 in the SM to its better timing parameters, e.g. smaller pore size (10 $\mu \mathrm{m}$ for the Photek 240 and $25 \mathrm{um}$ for the Photonis). The measured SPTR for the detectors are consistent with that statement. Another reason is the better anode timing uniformity for the Photek 240.

The measured TR is worse for SM detection than for minimum ionizing particle (MIP, Photek 240). But the TR is still at the level of 20-30 ps (compared with $\sim 16.6$ ps for both $120 \mathrm{GeV} / \mathrm{c}$ proton and not interacting in the SM positrons). The degradation of the time resolution probably could be explained in terms of the timing of the shower maximum development. We do not see significant difference in SM TR, obtained with Photek 240 at $12 \mathrm{GeV} / \mathrm{c}$ and $32 \mathrm{GeV} / \mathrm{c}$ beams. We also do not see big TR changes for different lead thickness in these measurements. To study this effect further, we plan to perform simulation of signal development in the SM with secondary emitters as an active layer.

The measured main parameters of the electromagnetic shower are well described $[8,9]$. The SM detector, $(1-100 \mathrm{GeV}$ electron energy) is usually located in the beginning of the shower, after less than $4 \mathrm{X}_{0}$ - absorber thickness. We used lead thickness in correspondence with the SM locations in described detectors. The transverse size of the shower is very small at such depth and allows good separation from other showers. Our SM detectors cover this transverse size for lead absorber. The presented results show that the TR is at the level of 20-30 ps. The SM based on the approach could be very economical. A sandwich type calorimeter based on the same principle (MCP for all active layers) is also possible to build, but the cost is still a question in such a design.

\section{CONCLUSION}

We made measurements with different types of MCP PMT as shower maximum detector. The measurements were performed at the Fermilab Test Beam Facility with $120 \mathrm{GeV} / \mathrm{c}$ primary proton beam and $12 \mathrm{GeV} / \mathrm{c}$ and $32 \mathrm{GeV} / \mathrm{c}$ secondary beams. We obtained time resolution for the SM detector based on Photek 240 at the level of $20-30$ ps. The SM detector, based on the Photonis MCP-PMT, also achieves a very good TR, $\sim 35$ ps. We feel that this level of 
performance, even with a large MCP pore size (diameter $\sim 25 \mu \mathrm{m}$ ), enables the development of SM detectors for collider experiments at a potentially much reduced cost. The success of the LAPPD project $[2,4]$ in developing affordable MCP's is encouraging in this respect. More savings are possible if it can be shown that bare MCP's without an associated photocathode, can give similar timing performance. We plan on performing a future beam test that can resolve this issue.

\section{Acknowledgment}

We would like to thank Henry Frisch for useful discussions, Mike Albrow for the sharing beam time and Aria Soha for the good beam delivery and control.

\section{References}

1. A. A. Derevshchikov, V. Yu. Khodyrev, V.I. Kryshkin, V.E. Rakhmatov, A. I. Ronzhin, "On possibility to make a new type of calorimeter: radiation resistant and fast". Preprint IFVE 90-99, Protvino, Russia, 1990.

2. O. H. W. Siegmund, J. B. McPhate, J. V. Vallegra, A. S. Tremsin, H. Frisch, J. Elam, A. Mane, R. Wagner, "Large Area Event Counting Detectors with High Spatial and Temporal Resolution" to be submitted to JINST; Dec, 2013.

3. A. Ronzhin, M. Albrow, M. Demarteau, S. Los, S. Malik, A. Pronko, E. Ramberg, A. Zatserklyaniy, "Development of a 10 ps level time of flight system in the Fermilab Test Beam Facility". Nuclear Instruments and Methods. A 623 (2010) 931.

4. psec.uchicago.edu/library/MCP_info/data_sheet_85011-501.pdf

5. J.Vav'ra, D.W.G.S. Leith, B. Ratcliff, E. Ramberg, M.Albrow, A. Ronzhin, C. Eartley, T. Natoly, E. May, K. Byrum. "Beam test of a Time-ofFlight detector prototype". Nuclear Instruments and Methods, A 606 (2009), 404.

6. S. Ritt. "Design and Performance of the $5 \mathrm{GHz}$ Waveform Digitizing Chip DRS4", http://drs.web.psi.ch/docs/nss08.pdf 
7. E. Ramberg, A. Ronzhin, A. Zatserklyaniy. "Waveform analysis of SiPM signals with DRS4 board". Physics Procedia, 37 (2012), 800 - 802. TIPP

8. A.I. Ronzhin, S.A. Akimenko, B.V. Chuiko, A.A. Derevshchikov, V.A. Kachanov, V.I. Kryshkin, A.S. Konstantinov, V.I. Lavrentev, V.I. Shelikhov, V.L. Solovyanov O.D. Tsai. "Study of EM shower maximum detector for the STAR at RHIC". Proceedings of the "Notre Dame 1993, Scintillating fiber detectors" 1993, 340-344.

9. S.A Akimenko, V.I Belousov, B.V Chujko, A.A Derevschikov, S.V Erin, V.A Kachanov, A.S Konstantinov, I.V Kotov, V.I Kryshkin, N.G Minaev, A.A Morozov, A.I Mysnik, V.L Solovianov, V.I Shelihov, K.E Shestermanov, O.D Tsai, A.N Vasiliev, A.I.Ronzhin. "Study of strip fiber prototype shower maximum detector for the STAR experiment at RHIC". Nuclear Instruments and Methods, A 365, (1995), 92-97. 